\title{
Myocardial Ischemia, Reperfusion and Free Radical Injury
}

\author{
Benedict R. Lucchesi, PhD, MD
}

\begin{abstract}
Reperfusion of coronary arteries to limit myocardial ischemic injury and extent of myocardlal necrosis is possible by either the use of fibrinolytic therapy, coronary angioplasty or coronary artery bypass surgery. The concept that early reperfusion may salvage jeopardized myocardium is derived from basic experimental studies which purported to demonstrate that the ultimate extent of irreversible myocardial injury could be reduced by reperfusion of the ischemic myocardium within 3 hours from the onset of regional myocardial ischemia. It is firmly established that salvage of ischemic myocardium is dependent on early restoration of blood flow to the myocardlum at risk. Desplte dependency on reoxygenation for ultimate survival, myocardial tissue that is reperfused and reoxygenated may be subjected to additional injurious insull due to reactive metabolites of oxygen. The cytotoxic species of oxygen are referred to as "oxygen free radicals." Coincident with the influx of inflammatory cells into the reperfused region is an additional loss of otherwise viable myocardial cells. There is strong support for the concept that the polymorphonuclear leukocyte is a contributor to the phenomenon of "reperfusion" or "reoxygenation" injury in the blood perfused heart. This discussion focuses on the role of the neutrophil as a potential contributor to the extension of tissue injury and reviews those interventions, which although in the experimental stage, offer promise of becoming therapeutically important in the future and may help elucidate the mechanisms undertying the potentially deleterious role of the neutrophil in situations involving whole blood reperfusion of the ischemic myocardium.
\end{abstract}

(Am J Cardiol 1990;65:14 I-23 I)

\footnotetext{
From the University of Michigan Medical School, Department of Pharmacology, Ann Arbor, Michigan. This study was supported by Grant HL-19782-1 1 from the National Heart, Lung, and Blood Institute, the National Institutes of Health, Bethesda, Maryland.

Address for reprints: Benedict R. Lucchesi, PhD, MD, The University of Michigan Medical School, Department of Pharmacology, M6322 Medical Science Building I, Ann Arbor, Michigan 48109-0626.
}

I is recognized that prolonged myocardial ischemia is accompanied by a time-dependent loss of the viability of myocardial cells in the jeopardized region of the heart. ${ }^{1}$ Reperfusion is necessary to initiate and maintain those functions responsible for reversal of the changes induced by ischemia and for the continued survival of the myocardial cells at risk of permanent damage. The development of pharmacologic methods for reperfusion of the ischemic heart has reduced mortality and has led to the acceptance of thrombolytic therapy as the standard approach to the management of patients with an evolving acute myocardial infarction. ${ }^{2}$

Reperfusion injury has been recognized as occurring in experimental models of myocardial ischemia. ${ }^{3}$ It has been assumed that the observed loss of cell viability associated with the reintroduction of oxygen was solely an acceleration of cell death in myocytes that were beyond the point of salvage, or had been injured irreversibly. Reexamination of this question suggests that reperfusion of the previously ischemic heart with oxygenated whole blood may be detrimental as well as beneficial to the myocyte. ${ }^{4-6}$ A significant effort has been devoted to gaining a better understanding of the oxygen paradox ${ }^{7}$ or the phenomenon of reperfusion injury, ${ }^{7,8}$ or both, which may be more appropriately referred to as reoxygenation injury (i.e., an extension in the loss of myocardial cell viability beyond that due to the ischemic process itself). A number of recent reviews have been devoted to an examination of the question of whether reactive species of oxygen, or oxygen free radicals, contribute to the development of irreversible myocardial cell injury during the period of reperfusion. ${ }^{7-10}$ The purpose of this essay is to focus attention on the role of the polymorphonuclear leukocyte $(\mathrm{PMN})$ as a contributor to the phenomenon of reperfusion injury and to call attention to factors that modulate the inflammatory response to myocardial cell injury.

\section{NEUTROPHIL-DEPENDENT DAMAGE TO THE ENDOTHELIUM AND CARDIAC MYOCYTE}

One of the important functions of the PMN is defense of the host organism. However, during altered physiologic states, the PMN may react in a manner that leads to tissue injury. The tissue damage resulting from myocardial ischemia activates a sequence of events characterized as an inflammatory response that occurs independently of any improvement in myocardial reoxygenation. The concept we propose is that the invading leukocytes and ensuing inflammatory response contributes to the extension of myocardial cell injury adding to the irreversible cell injury from the ischemia itself. Interventions directed against the inflammatory cells and the cytotoxic products produced at the site of myocardial injury may result in a 
reduction in the extent of tissue damage associated with myocardial ischemia or reperfusion, or both. The circulating PMN does not interact with the normal endothelial surface of the vasculature. However, the PMN can be activated in response to stimuli arising from the ischemic insult, either from within or outside of the vascular compartment, and can be induced to undergo shape changes and ultimately to adhere to the surface of the vascular endothelium. ${ }^{11}$

Infiltration of the ischemic myocardium by PMNs begins within 60 minutes of the onset of regional myocardial ischemia and increases progressively after the onset of reperfusion. ${ }^{12-14}$ Neutrophils invade the region of ischemic myocardium long before the transmural progression of irreversible injury reaches the subepicardial myocardial region. ${ }^{15}$ Reperfusion is essential to prevent further extension of ischemia-induced myocyte necrosis. In the absence of reperfusion, the slowly developing inflammatory response to tissue injury permits neutrophil-mediated injury to progress and requires 12 hours or more to become manifest by histologic examination of myocardial tissues. Factors involved in the initiation and termination of the myocardial inflammatory response are not understood in detail, but serve as an interesting target for the development of new therapeutic interventions to prevent extension of tissue injury, either during the ischemic period or shortly after reperfusion.

A number of studies ${ }^{9-16}$ have demonstrated that interventions that limit neutrophil function or interfere with the cytotoxic factors produced by the infiltrating phagocytic cells can reduce ultimate infarct size in experimental animals that have undergone a period of regional myocardial ischemia followed by reperfusion. The role of the neutrophil as a contributor to myocardial injury in response to ischemia was suggested initially in $1975 .{ }^{17}$ Direct studies have demonstrated that ultimate infarct size was reduced by $43 \%$ in the canine heart under conditions of neutropenia, compared with control animals subjected to 90 minutes of regional myocardial ischemia followed by reperfusion for 6 hours. ${ }^{5}$ This observation subsequently was confirmed by other investigators using alternative means of inducing neutropenia. ${ }^{6}$

Additional support for the concept of neutrophil-mediated myocardial injury is derived from the pharmacologic modulation of neutrophil function during myocardial ischemia and reperfusion. Recognition of the mechanism of protection provided by ibuprofen on experimental myocardial injury was an important step in addressing the role of the neutrophil in promoting reperfusion injury. Ibuprofen, in contrast to other inhibitors of cyclooxygenase, such as indomethacin and aspirin, ${ }^{18,19}$ possess myocardial protective properties without altering myocardial blood flow or the relationship between inyocardial oxygen supply and demand. ${ }^{20,21} \mathrm{~A}$ review of several studies indicated that of the nonsteroidal anti-inflammatory agents examined, only ibuprofen reduced neutrophil infiltration to the reperfused myocardium. This observation suggests that the PMN contributes to the extension of tissue injury on reperfusion ${ }^{5,6}$ and is in keeping with the original findings of Hill and Ward. ${ }^{17}$ Neither aspirin nor indomethacin reduced infarct size or the in vitro neutrophil respira- tory burst in response to stimulation of the PMN with $\mathrm{C}_{5 \mathrm{a}}$. Ibuprofen, however, prevented the complement induced activation of the PMN and the production of oxygen radicals. ${ }^{19}$ It is possible to conclude that ibuprofen, a nonsteroidal anti-inflammatory agent, does not exert its protective effect on the myocardium through inhibition of the cyclooxygenase pathway of arachidonic acid metabolism, or by altering factors that restore the balance between myocardial oxygen supply and demand.

Additional evidence for the role of the PMN as a mediator of reperfusion injury was obtained from studies in which prostacyclin, or a related analog, iloprost, as well as prostaglandin $E_{1}$, limited ultimate infarct size in a canine model of regional ischemia and reperfusion. The mechanism of action was attributed to the ability of the prostanoids to inhibit the formation and release of neutrophil-derived cytotoxic metabolites of oxygen.22 24 Evidence from a number of studies indicates that the pharmacologic inhibition of neutrophil function correlates with the observed beneficial effects on myocardial reperfusion injury.

\section{CHEMOTACTIC FACTORS AND THE ACTIVATION AND RECRUITMENT OF NEUTROPHILS}

The complement system serves as one of the most important sources of inflammatory mediators involved in the acute inflammatory response. Complement activation generates a potent neutrophil chemotactic factor, $\mathrm{C}_{5 \mathrm{a}}$. This anaphylatoxin has a role in the recruitment of PMNs to the site of tissue injury and in their activation. Proteolytic enzymes from the plasma and cellular sources react with individual complement components resulting in the localized formation of inflammatory mediators, without causing a generalized activation of the complement cascade. The presence of a tissue protease in the ischemic myocardium was suggested initially as being involved in cleaving the third component of complement into chemotactically active fragments that may stimulate the attraction of neutrophils to the myocardium. ${ }^{25,26} \mathrm{Car}-$ diac lymphatic drainage from the ischemic myocardium contains molecules of mitochondrial and subcellular origin that are bound to $\mathrm{Cl}_{\mathrm{q}}$, which may activate the complement cascade, promote the release of leukotactic anaphylatoxins, and stimulate the migration of PMNs to sites of tissue injury. ${ }^{27}$ Depletion of complement with cobra venom factor significantly reduced PMN infiltration into ischemic myocardium and resulted in a decrease in tissue injury associated with regional ischemia and reperfusion. ${ }^{25,28}$ Complement-derived products can exacerbate ischemic tissue injury and cardiac dysfunction through mechanisms that are independent of the presence of neutrophils. Neutrophil-independent injury by complement may be associated with the vascular actions of the $\mathrm{C}_{3 \mathrm{a}}, \mathrm{C}_{4 \mathrm{a}}$ and $\mathrm{C}_{5 \mathrm{a}}$, known to enhance vascular permeability and alter vessel tone. Furthermore, tissue injury may be a consequence of the formation of cytolytic membrane attack complex $\left(\mathrm{C}_{5 \mathrm{~b}-9}\right)$, which can result in the production of transmembrane channels and facilitate the influx of extracellular calcium and disruption of myocyte function. ${ }^{29}$ 


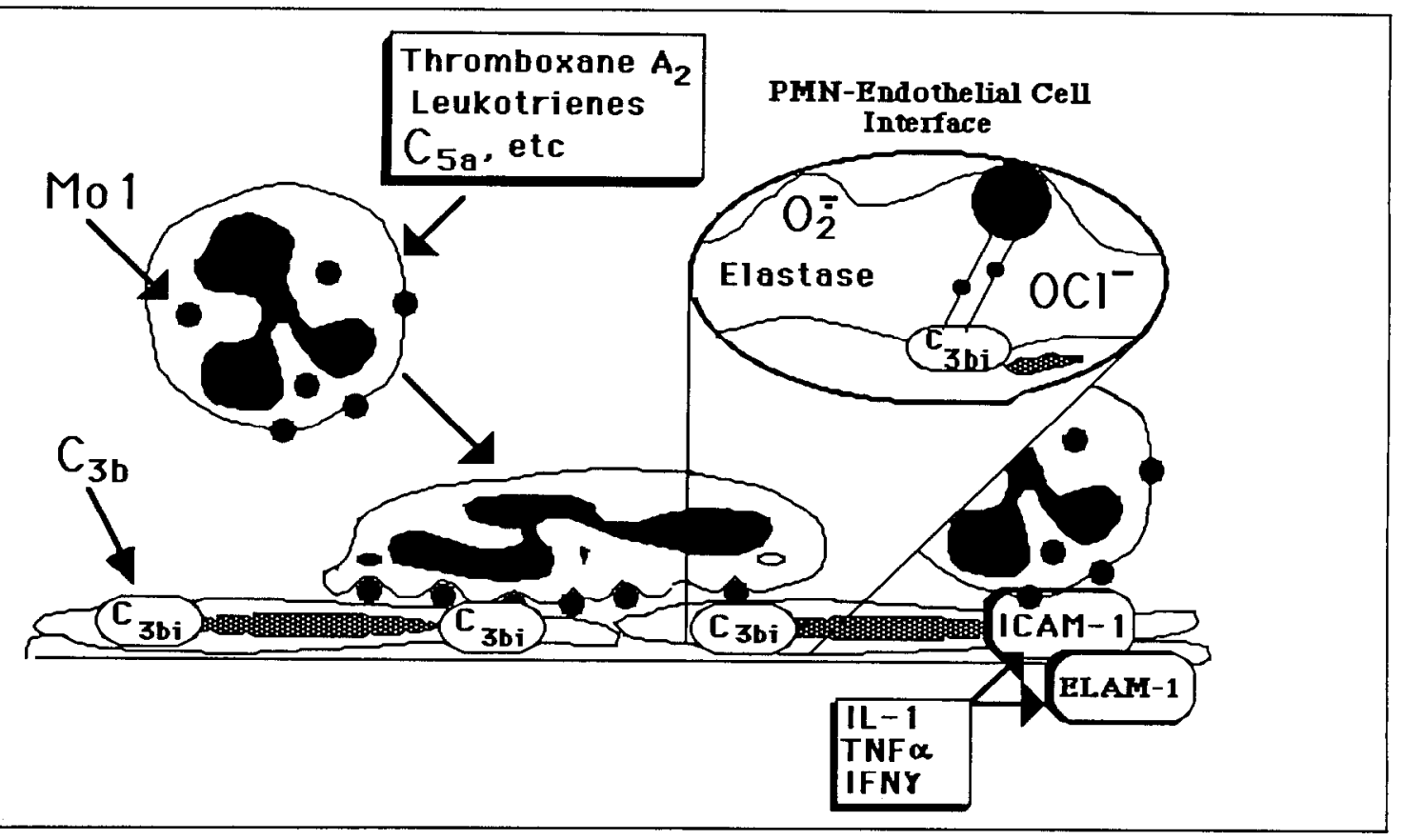

FIGURE 1. Representation of the leukocyte-endothelial cell interaction in response to injury. Endothelial injury secondary to ischemia and reoxygenation promotes neutrophil adherence to the cell surface. Endothelial cel surface molecules interact with neutrophil CD18 glycoprotein achesion-promoting receptor. $\mathbf{C}_{36 ;}$ derived from the activation of the complement system in response to injury serves to "opsonize" the endothelial cell and is the ligand for Mo1 expressed on the surface of the neutrophll. Expression of the neutrophil CD11b/CD18 heterodimeric complex (Mo1) on the plasma membrane of the neutrophil is a response to inflammatory stimul ( $\mathrm{C}_{5 a}$, leukotrienes, thromboxane, etc.). Neutrophil CD18 molecules can interact with intracellular adhesion molecule-1 (ICAM-1) on the endothelial cell membrane thereby serving as an alternate mechanism for the attachment of inflammatory cells to a target tissure. ICAM-1 and ELAM-1 (endothelial leukocyte adhesion molecule) are expressed in response to a variety of cytokines acting on the endothellal cell. In addition, the cytokines, interleukin-1 (IL-1), tumor necrosis factor (TNF) and interferon $\gamma$ (IFN $\gamma$ ) will induce synthesis and secretion of a neutrophil chemotactic factor by endothelial cells, thus permitting the endothelium to participate actively in the recruitment and attachment of inflammatory cell mediators to the tissue that has been "targeted" as a result of injury. Whether the sequence of events described occurs in the heart that has been subjected to ischemia and reperfusion remains a lopic of further study.

Endothelial cells may be an important source of a specific neutrophil chemotactic factor. ${ }^{30}$ Either interleukin-1 (IL-1) or tumor necrosis factor $\alpha$ (TNF- $\alpha$ ) will induce, in endothelial cells, the synthesis and secretion of a neutrophil chemotactic factor similar in structure to a human monocyte-derived neutrophil-activating factor. Neutrophil-activating factor will promote neutrophil chemotaxis and, in addition, will induce an increase in intracellular calcium, an oxidative burst, and granule exocytosis through a guanosine triphosphate-binding proteindependent pathway. Similar responses are observed with other chemotactic peptides (e.g., $\mathrm{C}_{5 \mathrm{a}}$, formyl-methionylleucyl-phenylalanine). ${ }^{31}$ The role that endothelial cellderived mediators may play in activating neutrophils after ischemic injury has not been investigated. These relationships are important in determining the mechanisms that initiate the activation of circulating neutrophils and promote their adherence to the injured vascular wall.

\section{ADHERENCE-PROMOTING CELL SURFACE GLYCOPROTEIN COMPLEXES AND THE INTERACTION BETWEEN THE GRANULOCYTES AND ENDOTHELIAL CELLS}

Granulocytes migrate across the vascular endothelium into the extravascular space in response to tissue injury. Initially, the inflammatory cells adhere to the vascular endothelial cell. A family of heterodimeric glycoproteins, possessing a common $\beta$ subunit of $95 \mathrm{kDa}$ (CD18) associated noncovalently with separate $\alpha$ subunits of 177,165 and $150 \mathrm{kDa}$, are present on the neutrophil. The neutrophil surface glycoproteins are designated as lymphocyte function-associated antigen-1 (LFA-1) (CD11a), Mol or Mac-1 (CD11b) and gp150,95 (CD11c), respectively. The Mol glycoprotein heterodimer is involved in the process of cellular adhesion and serves as the receptor for C3bi opsonized particles.

Distinct stimuli mobilize Mol from intracellular sites and lead to its expression on the cell surface. Mol participates in adherence of the leukocyte to the endothelial cell and to other PMNs, as well as in chemotaxis and spreading on cell surfaces. An increase up to twofold in Mol cell-surface expression has been observed. At least $50 \%$ of the total population of Mol receptors is located intracellularly. The increased expression of Mol increases neutrophil adherence to cell surfaces and facilitates chemotaxis. The increase in Mol glycoprotein expression is coupled to the release of neutrophil gelatinase which participates in the degradation of connective tissue, thus allowing for the passage of neutrophils through capillary walls and into the extravascular space. The up-rcgulation 
of Mol, for which C3bi serves as the ligand, would allow for enhanced recognition and attachment to endothelial surfaces that have been "opsonized." Injury to the endothelium from ischemia and reoxygenation promotes neutrophil adherence to the cell surface. There is enhanced binding of neutrophils to endothelial surfaces secondary to the interaction of neutrophil CD18 molecules with the intercellular adhesion molecule-1 (ICAM-1) on the endothelial cell membrane. ${ }^{32}$ Enhanced ICAM-1 expression on the endothelial cell surface can be induced by multiple inflammatory mediators including bacterial endotoxin and the cytokines IL-1, interferon- $\gamma$, and TNF$\alpha{ }^{33}$ A distinct endothelial leukocyte adhesion molecule (ELAM-1) that promotes neutrophil binding to the endothelium can be induced by specific cytokines. ${ }^{34}$ Whether a similar increase in the expression of ELAM-1, ICAM-1 or ICAM-1-like molecules occurs in ischemic endothelium is not known, but may play an important role in leukocyte localization to ischemic myocardium (Fig. 1).

It has been observed that specific cytokines will induce synthesis and secretion of a neutrophil chemotactic factor by endothelial cells. This observation and studies cited provide further evidence for an active role of the endothelial cell in modulating neutrophil activation and recruitment to ischemic tissues, rather than a "passive" participant in the interaction of inflammatory cells with the vascular wall. The interaction between endothelial cells and PMNs is required for an effective chemotactic response and for some forms of neutrophil-mediated vascular injury. This represents an important initial step in the sequence of events associated with the acute inflammatory response. A variety of agonists involved in acute inflammation, including thrombin, platelet-activating factor and leukotriene $\mathrm{C}_{4}$, are known to facilitate the adherence of neutrophils to the endothelial surface.

The neutrophil CD18 glycoprotein molecules may participate in other functions beside cell adhesion and chemotaxis. The glycoproteins may be important in the transduction of signals from the cell surface to intracellular enzymatic regulatory systems as suggested by the presence of an extensive intracellular domain providing sites for interaction with cytoplasmic proteins and cytoskeletal elements.

Monoclonal antibodies, specific for either the $\alpha$ or $\beta$ subunits of Mol, inhibit certain neutrophil/monocyte functions, such as the binding of C3bi-opsonized particles, and adhesive interactions of neutrophils and monocytes, including neutrophil aggrcgation, monocytc/ncutrophil adhesion and spreading on substrates (including vascular endothelium), and chemotaxis. The leukocytes of patients who are genetically deficient in their expression of the Mol glycoprotein exhibit an impairment of C3bi-particle binding and leukocyte adhesive interactions. The Mol glycoprotein has a domain that functions as the plasma membrane receptor for C3bi(CR3). The critical role of neutrophil glycoprotein adhesive receptors Mo1 in the inflammatory response of activated neutrophils is illustrated by the inhibitory effect of specific monoclonal antibodies on neutrophil-mediated endothelial cell injury in a rat lung model of adult respiratory distress syndrome ${ }^{35}$ and neutrophil-dependent vascular injury in skin. ${ }^{36}$

A monoclonal antibody (904) that binds to the Mol leukocyte cell adhesion-promoting glycoprotein CD11b/ CD18 was administered to open-chest anesthetized dogs 45 minutes after the induction of regional myocardial ischemia. Ischemia was produced by occluding the left circumflex coronary artery for 90 minutes followed by reperfusion for 6 hours. There was no difference between control and antibody-treated groups with respect to arterial blood pressure, heart rate or coronary blood flow. Administration of antibody produced no observable effect on circulating neutrophil counts demonstrating that antibody-bound neutrophils were not cleared from the circulation. Myocardial infarct size, expressed as a percentage of the area at risk of infarction, was reduced by $46 \%$ with anti-Mol treatment. The area at risk of infarction was similar between groups. Accumulation of neutrophils within the myocardium was reduced significantly with anti-Mol treatment. ${ }^{37,38}$ Similar protective effects were obtained in subsequent studies with anti-Mol $\mathrm{F}\left(\mathrm{ab}^{\prime}\right) 2$ fragments in which antibody treatment was maintained for 48 hours into the reperfusion period and infarct size was determined at 72 hours after reperfusion. Figure 2 summarizes the results from studies in which treatment with anti-Mol antibody was associated with a significant protective effect regardless of whether infarct size was determined after 6 or 72 hours of reperfusion. Furthermore, regional myocardial blood flow determinations indicated that both groups had equal degrees of ischemia in the inner two-thirds of the myocardium during the period of coronary artery occlusion. Results with the anti-Mol antibody indicated that the adhesive interactions of the neutrophil played a central role in neutrophil-mediated damage and that inhibition of neutrophil adhesive interactions can reduce the extent of irreversible myocardial damage associated with reperfusion. The results are in accord with earlier studies in which myocardial reperfusion injury was reduced by a variety of interventions that shared one common property; i.e., they altered the ability of the neutrophil to react with its target tissue, the ischemically altered myocardial cell. ${ }^{24,39-41}$

The relative contribution of Mo1, LFA-1 and p150,95 to leukocyte endothelial adhesion is dependent on the applied stimulus and the particular inflammatory cell involved. Monocytes and granulocytes employ distinct subunits of the adhesion-promoting glycoproteins when they interact with the vascular endothelium. Mo1, as well as LFA-1 and p150,95, molecules have been shown to be involved in leukocyte-endothelial cell adhesion. The adhesion may be prevented by antibodies directed against the specific portion of the heterodimeric surface molecules. ${ }^{42}$ Granulocyte adhesion to endothelial cells, by means of the CD11/CD18 subunits, varies according to the activation state of the granulocyte and endothelial cell. It is reported that granulocyte adhesion, promoted by activation of the inflammatory cells with PMA, was mediated primarily by Mol and that the adhesion was not inhibited by antibodies directed against LFA-1 or p150,95. Exposure of endothelial cells to recombinant 
I.ONG-TERM PROTECTIVE EFFECT OF

ANTI-Mo1 ANTIBODY ON MYOCARDIAL INFARCT SIZE
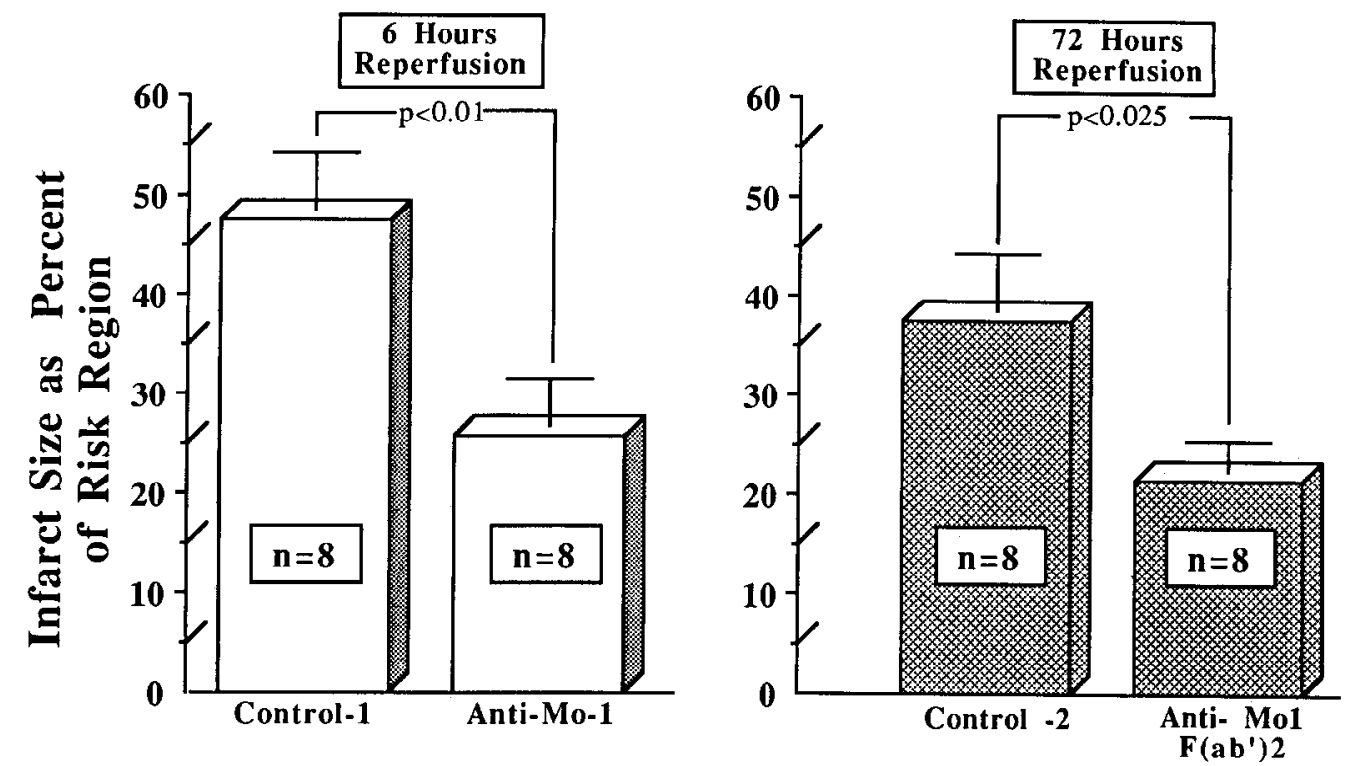

FIGURE 2. Myocardial infarct size in the canine heart expressed as a percentage of the risk region determined in 2 studies, each incorporating a control group and a group of animals treated with a murine monoclonal anti-Mo1 antibody. The graph on the left represents the results from a study in which coronary artery occlusion was maintained for 90 minutes followed by reper fusion for 6 hours, after which infarct size was determined. The treatment consisted of the administration of $1 \mathrm{mg} / \mathrm{kg}$, intravenously, of Mol antibody given over 10 minutes, starting 45 minutes after occlusion of the left circumflex coronary artery. Inhibition of the Mo1 receptor by the anti-Mo1 antibody treatment resulted in a $46 \%$ reduction in myocardial infarct size compared with a placebo-treated group. To determine if the findings of the 6-hour study represented delay of infarction as opposed to prevention of irreversible injury, a second study was performed in which infarct size was determined 72 hours after repertusion. The graph on the right represents data obtained in a study in which the F(ab'2) fragment of the Mo1 antibody was used in a dosing regimen of $1 \mathrm{mg} / \mathrm{kg}$ over 5 minutes, starting 45 minutes after coronary artery occlusion with repeated $0.5 \mathrm{mg} / \mathrm{kg}$ doses at 12, 24, 36 and 48 hours after repertusion. Due to the relatively short half-ifie of the $F\left(a b^{\prime 2}\right)$ fragment, repeated doses were necessary to modulate neutrophil function throughout the first $\mathbf{4 8}$ hours of the reperfusion period. Infarct size was determined 24 hours after the last dose of the Mo1 antibody (72 hours after reperfusion). Despite the withdrawal of the antibody treatment, infarct size was reduced when determined at 72 hours after reperfusion. The data suggested that a sustained reduction in infarct size can be achieved by the modulation of neutrophil reactivity.

IL-1 permitted each of the subunits to contribute to granulocyte adhesion. ${ }^{42}$ Current data suggest that the $\alpha$ subunit of each of the 3 heterodimers contains a common region in the extracytoplasmic domain which mediates the interaction of the leukocyte with the endothelial cell or with the subendothelial structures during transmigration of leukocytes to regions of tissue injury and inflammation. ${ }^{42}$

The data demonstrate that inhibition of neutrophil adhesion reduced the extent of myocardial reperfusion injury and are in keeping with the proposition that neutrophils serve an important role as mediators of inflammation, extending myocardial injury beyond that caused by the ischemic process itself. The data also support the hypothesis that a significant number of myocardial cells at risk are viable after a 90 -minute ischemic period, but then progress to an irreversible state of injury on reperfusion with the continued accumulation of PMNs. Studies from this laboratory ${ }^{43}$ have identified a specific "time window" in which neutrophils participate in the extension of myocardial infarction after ischemia and reperfusion. The inhibition of neutrophil activation and accumulation in the myocardium over a prolonged period of time ( $>48$ hours) is necessary to ensure a beneficial effect on the ultimate extension of irreversible myocardial injury. Interventions that have limited pharmacologic half-lives, while effective over brief periods of observation, may not show protective benefits if given in a single initial dose when infarct size is assessed at a period far removed from the point of administration of the treatment under study. Consideration must be given to the pharmacokinetic properties of selected interventions along with the dynamic and progressive nature of myocyte injury. The failure to consider the time course of events in myocardial ischemic and reperfusion injury may account for the discordant results in published reports. To date, published reports have failed to document a consistent progression of cellular injury attributable to the period of reperfusion and distinct from the progressive cell death entirely attributable to the period of ischemia.

\section{NEUTROPHILS, OXYGEN RADICALS, MYOCARDIAL INJURY AND PROTECTION BY FREE RADICAL SCAVENGERS}

Under some conditions of regional myocardial ischemia and reperfusion, neutrophils adhere to the vessel 
wall at sites of inflammation and release toxic products capable of damaging the adjacent endothelium and myocytes within the reperfused region. It is recognized that neutrophils can release cytotoxic products extracellularly without undergoing destruction themselves. Neutrophils can release a variety of mediators capable of promoting tissue injury, including proteolytic enzymes, platelet-activating factor, arachidonic acid metabolites and activated species of oxygen. The last mentioned group of neutrophil-derived products, superoxide anion $\left(\mathrm{O}_{-2}\right)$, hydrogen peroxide $\left(\mathrm{H}_{2} \mathrm{O}_{2}\right)$, hydroxyl anion $(\cdot \mathrm{OH})$ and hypochlorous anion $(\mathrm{OCl}-)$ are significant cytotoxic products derived from the metabolism of molecular oxygen on perturbation of the PMN. The generation of cytotoxic metabolites of oxygen, generated within the microenvironment formed between the adherent activated PMNs and altered endothelial cells, leads to an increase in vascular permeability and myocyte damage. This situation is "explosive" on reperfusion as neutrophils are directed and attracted to the reperfused region under the influence of the local accumulation of chemoattractants. During an extended period of regional ischemia of $\geq 40$ minutes, ${ }^{14}$ the stage is set for the neutrophil-mediated inflammatory response and injury that involves normal or reversibly injured cells and is accelerated by reperfusion of the ischemic region. Reperfusion injury, therefore, involves cells that were viable or recoverable up until the time that reperfusion was instituted. The extension of cell death, on reperfusion of the ischemic myocardium, is mediated by the neutrophil-derived cytotoxic products of oxygen metabolism. In the absence of reperfusion, all cells that are ischemic for a sufficiently long period will undergo irreversible changes, and reperfusion is essential for survival of the myocytes that are viable up until the time of reperfusion. However, a portion of the still viable myocardial cells will undergo further injury due to the cytotoxic actions of the reactive species of oxygen.

Jolly et al ${ }^{44}$ were the first to provide evidence for the in vivo participation of oxygen radicals in myocardial reperfusion injury. They demonstrated that the administration of superoxide dismutase (SOD) plus catalase to anesthetized dogs, subjected to a 90-minute interval of regional myocardial ischemia followed by reperfusion, reduced ultimate infarct size when the scavenger enzymes were administered either before the induction of regional myocardial ischemia or 15 minutes before myocardial reperfusion. However, the enzymes were without beneficial cffect if thcy were infused $\mathbf{4 0}$ minutes after reperfusion had been instituted. The data suggested that an extension of myocardial injury, in addition to that associated with the ischemia-induced myocardial cell death, occurred very early in reperfusion, and myocyte damage was attenuated by pretreatment with appropriate antioxidants. Subsequent studies have demonstrated that SOD, without the addition of catalase, was equally effective in preventing the extension of myocardial injury, thereby implicating superoxide anion as the primary mediator of the myocardial damage associated with reperfusion. The relatively short pharmacologic half-life (6 to 10 minutes) of SOD is a drawback to the study of the scavenger in experimental protocols that are extended several days after reperfusion. Neutrophil chemoattraction to the ischemically injured myocardium and the local formation of neutrophil-derived reactive species of oxygen will continue to effect a cytotoxic influence on the viable myocardial cells in the reperfused tissue. The free radical-induced injury is initiated on reperfusion and continues until mechanisms, still to be elucidated, bring about a cessation of the inflammatory response.

Disagreement regarding the cardioprotective role of SOD may relate to the use of different durations for induction of ischemic injury and reperfusion. To investigate this concept we undertook studies that used a modified form of SOD in which the enzyme was conjugated to polyethylene glycol (PEG-SOD). ${ }^{45}$ The conjugated form of SOD has a pharmacologic half-life of 30 hours. Significant plasma SOD activity was demonstrated in the dog up to 7 days after a single intravenous administration of $1,000 \mathrm{U} / \mathrm{kg}$ of PEG-SOD. Two protocols, differing in the mode of administration and duration of the reperfusion interval, were used. Dogs were subjected to occlusion of the circumflex coronary artery for 90 minutes, then reperfused for either 6 hours (protocol A) or for 4 days (protocol B). The dogs received either PEG conjugated to albumin (PEG-ALB) or PEG-SOD, 1,000 U/ kg. In protocol A, treatment was administered starting 15 minutes before coronary artery occlusion and continued for 2 hours, terminating 15 minutes after reperfusion. Infarct size was determined 6 hours later. In protocol B, the conjugated proteins were given 15 minutes before reperfusion and ended simultaneously with reperfusion. Infarct size was measured after 4 days. A significant reduction in myocardial infarct size, expressed as a percent of the area at risk, was observed in protocol A in the group of dogs that received PEG-SOD compared with the PEGALB-treated controls. Likewise, in the extended protocol, in which infarct size was determined 4 days after reperfusion, the PEG-SOD-treated group had a significant reduction in ultimate infarct size (Fig. 3). Hemodynamic variables did not differ during the period of coronary artery occlusion. The respective collateral blood flows to the inner two-thirds of ischemic myocardium, determined 60 minutes after occlusion, did not differ between the groups. Infarct size was inversely related to collateral blood flow in the PEG-ALB-treated group. The regression line describing the relationship between the extent of irreversible myocardial injury and collateral blood flow was shifted downward (analysis of covariance, $p=0.01$ ) in the group of dogs treated with PEG-SOD. With use of the mean collateral blood flow to the inner two-thirds of the ischemic myocardium as a covariate, infarct size, expressed as a percentage of the area at risk, was smaller for the PEG-SOD-treated group than for the PEG-ALB-treated group ( $p<0.05$ ). The data demonstrated that PEG-SOD achieved a reduction in ultimate infarct size when quantitation of myocardial injury was performed after 4 days of reperfusion, a time when the plasma SOD activity was maintained well above that found in the PEG-ALB control group. These data are consistent with studies cited which demonstrated the necessity to suppress neutrophil function up to 48 hours to effect and maintain a reduction in infarct size determined 
SUSTAINED PROTECTIVE EFFECT OF PEG-SOD ON MYOCARDIAL INFARCT SIZE
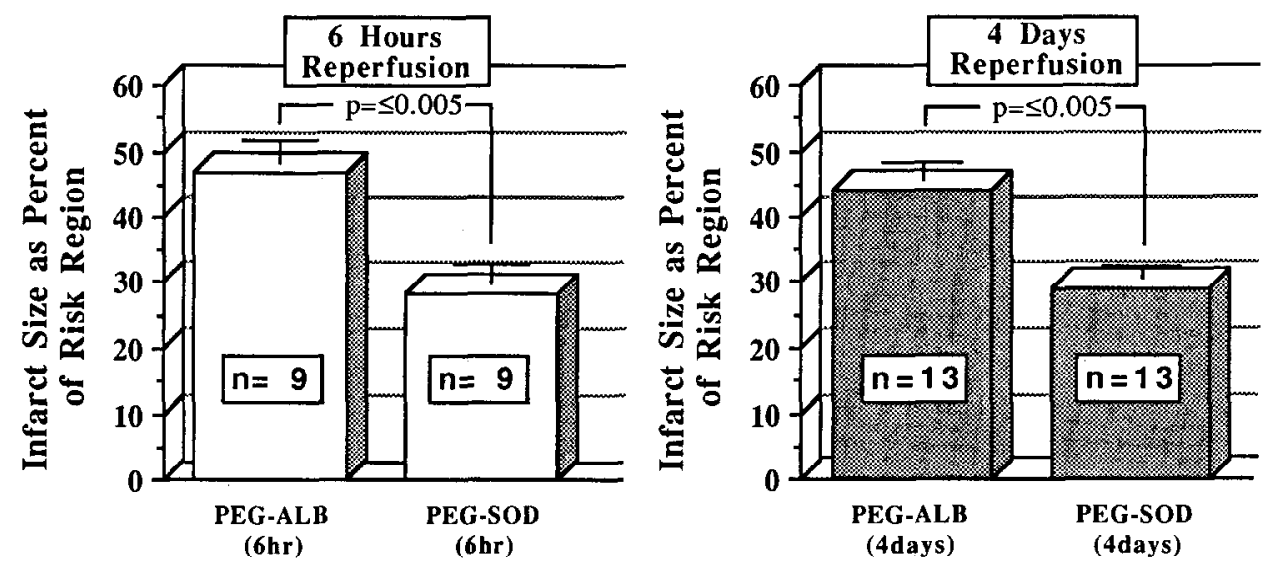

FIGURE 3. The effect of polyethylene-glycol conjugated superoxide dismutase (PEG-SOD) on myocardial infarct size due to a 90-minute occlusion and exbsequent reperfusion of the leff circumflex coronary artery in the canine heart. A single dose of PEGSOD of 1,000 U/kg was administered before reperfusion in each of the 2 studies. The control groups received PEG conjugated to alloumin (PEG-ALB) so that the edministered amount of PEG and conjugated protein was comparable in all dogs. Infarct size In the 2 groups of dogs, depicted on the left, was determined after 6 hours of reperfusion. The 2 groups of dogs shown on the right had inferet measurements obtained 4 days after reperfusion. The data illustrated that the PEG-SOD with a 30 -hour plasma half-tife is able to provide sustained protection against the cytotoxic effects of superoxide anion dorived in part from the infitration of inflammatory cells into the reperfused myocardium.

at 72 hours. ${ }^{43}$ These observations support the hypothesis that a critical "time window" exists during which antineutrophil or antioxidant therapy, or both, must be maintained to effect a reduction in the ultimate extent of the infarcted myocardium after periods of ischemia and reperfusion. PEG-SOD may have efficacy over that of the native enzyme because of its continued presence in the circulation and its ability to adhere to the surface of the vascular endothelium. PEG conjugation of SOD enhances cell association and uptake of the enzyme in a manner that renders the vascular endothelium more oxidant resistant.

The vulnerability of the endothelium to injury from reactive species of oxygen may be related to a number of factors, which include anatomic proximity to activated and invading inflammatory cells and localization of xanthine oxidase activity within endothelial cells. Vascular endothelial cells subjected to an ischemic equivalent of anoxic incubation followed by reoxygenation become effective generators of oxygen free radicals as determined by electron paramagnetic resonance measurements with a spin trap agent, 5,5-dimethyl-1-1-pyrroline-1-oxide. ${ }^{46}$ SOD or catalase abolished the radical signal, suggesting that oxygen is sequentially reduced from $\mathrm{O}_{2}$ to $\mathrm{H}_{2} \mathrm{O}_{2}$ to .OH. Attenuation of the free radical signal by oxypurinol suggested that xanthine oxidase was a major source of the superoxide anion. It is conceivable that on reperfusion of the ischemic myocardium, the endothelial cells, as well as the adherent and infiltrating neutrophils, may provide the initial source of cytotoxic species of reduced oxygen and that the subsequent inflammatory process, involving the continued migration and accumulation of PMNs to the injured myocardium, provides a sustained source of oxygen radicals that diminish with time as reparative processes occur.

Interpretation of data based on inhibition of free radical production by pharmacologic inhibition of xanthine oxidase are cautioned. Recent data suggest that the rate of conversion of the dehydrogenase form of the enzyme to the oxidase form, in the rat heart, is too slow to account for the observed results. ${ }^{47}$ It has been reported that allopurinol scavenges $\cdot \mathrm{OH}$ at rates comparable to those of mannitol. ${ }^{48}$ Oxypurinol, the active metabolite of allopurinol, is more effective as a scavenger of $\cdot \mathrm{OH}$. Time-dependent formation of the active metabolite in animals pretreated with allopurinol may explain the failure of some investigators ${ }^{49}$ to observe a protection of the ischemic myocardium when the drug was evaluated acutely, as opposed to studies in which a pretreatment regimen was used permitting accumulation of the metabolite, oxypurinol, in which a reduction in ultimate infarct sizc was reported..$^{50,51}$ Furthermore, oxypurinol combines with the myeloperoxidase-derived oxidant, hypochlorous acid, preventing the latter from inactivating $\alpha_{1}$-protease inhibitor and thereby protecting inflamed tissues from the damaging effects of elastase. ${ }^{48}$

\section{OTHER FREE RADICAL SCAVENGERS IN THE PREVENTION OF MYOCARDIAL REPERFUSION INJURY}

Previous studies have shown that sulfhydryl-containing compounds, including mercaptoproprioyl glycine (MPG), are capable of reacting with free radicals and inhibiting their biologic effects. ${ }^{52,53}$ Mitsos et al ${ }^{54}$ report- 
ed the in vitro free radical-scavenging effects of MPG, as well as its potential to reduce myocardial infarct size in the canine heart subjected to 90 minutes of regional ischemia followed by 6 hours of reperfusion. The free radical scavenger was effective when administered intravenously just before the onset of reperfusion, suggesting that the sulfhydryl compound protects against that component of myocyte injury associated with reperfusion. Related observations regarding the cardioprotective effects of MPG have been provided by other investigators who have examined the phenomenon of myocardial stunning. It was noted that SOD, as well as MPG, were equally effective in restoring regional contractile function to the heart made ischemic for a brief period ( 15 minutes) followed by reperfusion. ${ }^{55,56}$ The administration of the scavengers was effective when given before the onset of ischemia or immediately before reperfusion. If administration was delayed after the start of reperfusion, no benefit was demonstrated. These findings are reminiscent of the initial studies with SOD ${ }^{4}$ in which salvage of myocardial tissue was accomplished only if the scavenger had been given before the onset of reperfusion.

The recognition that sulfhydryl-containing compounds could scavenge oxygen free radicals and provide a degree of protection against myocardial reperfusion injury and myocardial stunning led Westlin and Mullane ${ }^{57}$ to explore the cardioprotective actions of other sulfhydrylcontaining compounds. Several angiotensin-converting enzyme (ACE) inhibitors were examined for their ability to suppress superoxide anion formation in vitro and to improve postischemic cardiac function in vivo in a canine model of regional myocardial ischemia and reperfusion. Three sulfhydryl-containing ACE inhibitors, captopril, its stereoisomer SQ 14,534, and an analog, zofenopril (SQ 27,703) were compared with enalaprilat and teprotide. The latter 2 agents lack the sulfhydryl group, but inhibit ACE. Two additional sulfhydryl-containing compounds, MPG and $\mathrm{N}$-acetylcysteine (NAC), which are not ACE inhibitors, were studied for suppression of free radical formation in vitro. The autooxidation of epinephrine to adrenochrome is mediated by superoxide anions and was inhibited by captopril, SQ 14,534, and zofenopril, with similar $\mathrm{IC}_{50}$ values of 8 to $10 \mu \mathrm{M}$, but not enalaprilat or teprotide $\left(\mathrm{IC}_{50}\right.$ greater than $\left.1,000 \mu \mathrm{M}\right)$. The autooxidation reaction is inhibited as well by MPG and NAC. Captopril, MPG or NAC, but not teprotide or enalaprilat, were shown to scavenge superoxide anion production by the purine-xanthine oxidase reaction and by canine-activated neutrophils. Therefore, captopril scavenges superoxide anions in vitro independent of an action on ACE; an action that is most likely related to the presence of a sulfhydryl moiety. Pretreatment of dogs with captopril, intravenously $(5 \mathrm{mg} / \mathrm{kg}$ ), before occlusion of the coronary artery for 15 minutes followed by reperfusion resulted in a 40 to $60 \%$ return to active shortening within 60 minutes of reperfusion. However, equihypotensive doses of enalaprilat did not improve segmental function during reperfusion. Dogs given captopril immediately before restoring coronary blood flow had a similar return of function as that observed in dogs treated with the drug before occlusion. SQ 14,534, the isomer of cap- topril, which is 100-fold less potent as an ACE inhibitor, but equipotent in scavenging superoxide anions, also improved reperfusion-induced cardiac dysfunction when administered at reperfusion $(5 \mathrm{mg} / \mathrm{kg})$. Captopril improved postischemic contractile derangements by a mechanism independent of ACE inhibition. Restoration of blood supply to the ischemic myocardium provoked ventricular fibrillation in $37.5 \%$ of control dogs, but in only $9 \%$ of those administered enalaprilat and $0 \%$ of captopril-treated dogs. SQ 14,534 did not reduce the incidence of ventricular fibrillation ( $40 \%$ ), indicating that the antifibrillatory actions may be related to ACE inhibition, or to a direct effect of the free radical scavenger on the integrity of the coronary vascular endothelium and the preservation of regional coronary artery blood flow.

\section{CONCLUSIONS}

Regardless of the cellular site of generation (endothelial cell, neutrophil or other cellular loci), the role of reactive species of oxygen in mediating cellular injury associated with reperfusion has gained wide support through a number of studies that have been reviewed recently. ${ }^{58-61}$ These studies have not always been in agreement with the concept that reperfusion of the ischemic myocardium may result in an extension of injury beyond that which has resulted from the ischemic insult itself. We have concluded that the failure to obtain agreement among laboratories is related to differences in the models used with respect to the instrumentation of the heart in chronic animal studies, the duration of occlusion (40 vs 90 minutes), and the duration of reperfusion (6 hours vs 4 days). ${ }^{43}$ The pharmacokinetic properties of interventions used to modify the extent of reperfusion injury must be given special consideration because the neutrophil-mediated component of reperfusion injury may be operative for several days. ${ }^{46}$ Studies reporting negative effects with native SOD may be related to the short half-life of its free radical-scavenging capacity, which compromises the chances of observing a protective effect after 4 days of reperfusion. ${ }^{45}$ This concern is applicable to other interventions studied in animal models in which assessment of efficacy is made several days after cessation of the treatment regimen. Because the PMN is implicated as being a major contributor to the observed injury on reperfusion, it is essential that the applied stimulus (ischemia) required for the ultimate chemoattraction of the PMN to the reperfused region be of sufficient duration. Short ischemic intervals (40 minutes), as opposed to longer (90 minutes) times of ischemic injury, may not serve as a sufficient stimulus for neutrophil recruitment, ${ }^{14}$ thus making it unlikely that interventions designed to mitigate against the deleterious effects of neutrophil-dependent cytotoxic products will manifest their therapeutic effect. ${ }^{62}$ The time-dependent relationship between the duration of regional myocardial ischemia and the manifestation of reperfusion injury has been suggested by the study of Eng et $\mathrm{al}^{63}$ and must be taken into consideration in the design of an experimental protocol for the study of reperfusion injury and its modification by pharmacologic interventions. The ability of infarct expansion to develop late in the reperfusion period must 


\section{A SYMPOSIUM: THE RENIN-ANGIOTENSIN SYSTEM — TISSUE SPECIFIC ISSUES}

be recognized. ${ }^{64,65}$ The modulation of the extent of reperfusion injury with an intervention possessing a relatively short pharmacologic half-life in relation to the period of reperfusion must be appreciated in the protocol design. ${ }^{45}$

\section{REFERENCES}

1. Reimer KA, Lowe JE, Rasmussen MM, Jennings RB. The wavefront phenomenon of ischemic cell death. I: myocardial infarct size vs. duration of coronary occlusion in dogs. Circulation 1977;56:786-793.

2. Topol EJ. Advances in thrombolytic therapy for acute myocardial infarction. $J$ Clin Pharmacol 1987;26:735-745.

3. Hearse DJ, Humphrey SM, Nayler WG, Slade A, Border D. Ultrastructural damage associated with reoxygenation of the anoxic myocardium. $J \mathrm{Mol} \mathrm{Cell}$ Cardiol 1975:5:315-324.

4. Jolly SR, Kane WJ, Bailie MB, Abrams GD, Lucchesi BR. Canine myocardial reperfusion injury: its reduction by the combined administration of superoxide dismutase and catalase. Circ Res 1984:54:277 285

5. Romson JL, Hook BG, Kunkel SL, Abrams GD, Schork MA, Lucchesi BR. Reduction of the extent of ischemic myocardial injury by neutrophil depletion in the dog. Circulation 1983;67:1016-1023.

6. Mullane KM, Read N, Salmon JA, Moncada S. Role for leukocytes in acute myocardial infarction in anesthetized dogs: relationship to myocardial salvage by antiinflammatory drugs. $J$ Pharm Exp Ther 1984;228:510-552.

7. Hess ML, Manson NH. Molecular oxygen: friend and foe. The role of the oxygen free radical system in the calcium paradox, and oxygen paradox and ischemia/reperfusion injury. $J$ Mol Cell Cardiol 1984:16:969-985.

8. McCord JM. Oxygen-derived free radicals in post ischemic tissue injury. $N$ Engl J Med 1985;312:159-163.

9. Lucchesi BR, Mullane KM. Leukocytes and ischemia-induced myocardial injury. Ann Rev Pharmacol Toxicol 1986;26:201-224.

10. Werns $S W$, Lucchesi BR. Leukocytes, oxygen radicals and myocardial injury due to ischemia and reperfusion. Free Radicals Biol Med 1988:4:3l-37.

11. Bevilacqua MO, Poker JS, Mendrick DL, Cotran RS, Gimbrone MA Jr. Identification of an inducible endothelial leukocyte adhesion molecule, ELAM-1. Proc Natl Acad Sci 1987;84:9238-9242.

12. Sommers HM, Jennings RB. Experimental acute myocardial infarction: histologic and histochemical studies of early myocardial infarcts induced by temporary or permanent occlusion of a coronary artery. Lab Invest 1964;13:1491 1503 .

13. Engler RL, Dahgren MD, Peterson MA, Dobbs A, Schmid-Schonbein GW. Accumulation of polymorphonuclear leukocytes during 3-h experimental ischemia. Am J Physiol 1986;251:H93-HIO0.

14. Go LO, Murry CE, Richard VJ, Weischedel GR, Jennings RB, Reimer KA. Myocardial neutrophil accumulation during reperfusion and reversible or irreversible ischemic injury. Am J Physiol 1988:255:H1188-H1198.

15. Jennings RB, Reimer KA. Factors involved in salvaging ischemic myocardium: effect of reperfusion of arterial blood. Circulation 1984;68:suppl 1:125-136. 16. Werns SW, Lucchesi BR. Myocardial ischemia and reperfusion: the role of oxygen radicals in tissue injury. Cardiovasc Drugs Ther 1989;2:761-769.

17. Hill $\mathrm{JH}$, Ward PA. The phlogistic role of $\mathrm{C} 3$ leukotactic fragments in myocardial infarcts of rats. J Exp Med 1975;133:885-900

18. Jugdutt BI, Hutchins GM, Bulkley BH, Pitt B, Becker LC. Effect of indomethacin on collatera! blood flow and infarct size in the conscious dog. Circulation 1979:59:734-743.

19. Flynn PJ, Becker WK, Vercellotti GM, Weisdorf DJ, Craddock PR, Hammerschmidt DE, Lillehei RC, Jacolb H. Ibuprofen inhibits granulocyte responses to inflammatory mediators. Inflammation 1984:8:33-44.

20. Jugdutt BI, Hutchin GM, Bulkley BH, Becker LC. Salvage of ischemic myocardium by ibuprofen during infarction in the conscious dog. Am J Cardiol $1980,46: 74-82$

21. Romson JL, Hook BG, Rigot VH, Schork MA, Swanson DP, Lucchesi BR. The effect of ibuprofen on accumulation of indium-111-labeled platelets and leukocytes in experimental myocardial infarction. Circulation 1982.666:10021011 .

22. Simpson PJ, Mitsos SE, Ventura A, Gallagher KP, Fantone JC, Abrams GC, Schork MA, Lucchesi BR. Prostacyclin protects ischemic-reperfused myocardium in the dog by inhibition of neutrophil activation. Am Heart J 1987;113:129137.

23. Simpson PJ, Mickelson JK, Fantone JC, Gallagher KP, Lucchesi BR. Iloprost inhibits ncutrophil function in vitro and limits expcrimental infaret size in canine heart. Circ Res 1987,60:666-673.

24. Simpson PJ, Mickelson J, Fantone JC, Gallagher KP, Lucchesi BR. Reduction of experimental canine myocardial infarct size with prostaglandin $E_{1}$ : inhibition of neutrophil migration and activation. $J$ Pharm Exp Ther 1988;244:619 624 .

25. Hill JH, Ward PA. The phlogistic role of $\mathrm{C} 3$ leukotactic fragments in myocardial infarcts of rats. J Exp Med 1975:133:885-400.

26. Hill JH Ward PA. C3 leukotactic factors produced by a tissue protease. $J$ Exp Med 1969:130:505-518.

27. Rossen RD, Michael LH, Kagiyama A, Savage HE, Hanson G, Reisberg
MA, Moake JN, Kim SH, Self D, Giannini E, Entman ML. Mechanism of complement activation after coronary artery occlusion. Evidence that myocardiat ischemia in dogs causes release of constituents of myocardial subcellular origin that complex with human $\mathrm{Clq}$ in vivo. Circ Res 1988,62:572-584. 28. Crawford MH, Grover FL, Kolb WP, McMahan A, O'Rourke RA, McManus LM, Pinckard RN. Complement and neutrophil activation in the pathogenesis of ischemic myocardial injury. Circulation 1988:78:1449. 1458. 29. Muller-Eberhard HJ. Complement: chemistry and pathways. In: Gallin Jl, Goldstein R, Synderman R, eds. Inflammation: Basic Principles and Clinical Correlates. New York: Raven Press, 1988:21-53.

30. Strieter RM, Kunkel SL, Showel HJ, Marks RM. Monokine induced gene expression of a human endothelial cell-derived neutrophil chemotactic factor. Biochem Biophys Res Comm 1988,156:1340-1345.

31. Thelen M, Peveri $P$, Kernen $P$, Tscharner VV, Walz A, Baggiolini $M$ Mechanism of neutrophil activation by NAF, a novel monocyte-derived peptide agonist. FASEB J 1988;2:2702-2706.

32. Smith CW, Rothlein R, Hughes BJ, Mariscalco MM, Rudloff HE, Schmal stic FC, Anderson DC. Recognition of an endothelial determinant for CD-18 dependent human neutrophil adherence and transendothelial migration. I Clin Invest 1988:82:1746-1756

33. Prober JS. Cytokine-mediated activation of vascular endothelium. Physiology and pathology. Am J Pathol 1988:133:426-433.

34. Bevilacqua MO, Poker JS, Mendrick DL, Cotran RS, Gimbrone MA Jr. Identification of an inducible endothelial leukocyte adhesion molecule, ELAM-1. Proc Natl Acad Sci 1987,84:9238-9242.

35. Ismail G, Morganroth ML, Todd RF III, Boxer LA. Prevention of pulmonary injury in isolated perfused rat lungs by activated human neutrophils preincubated with anti-Mol monoclonal antibody. Blood 1987,69:1167-1174

36. Anfors K, Lundberg C, Lindbom L, Lunderg K, Beaty PG, Harlan JM. A monoclonal antibody to membrane glycoprotein complex CD 18 inhibits polymor phonuclear leukocyte accumulation and plasma leakage in vivo. Blood 1987,69. $338-340$.

37. Simpson PJ, Todd RF III, Fantone JC, Mickelson JK, Griffin JD, Lucchesi BR. Reduction of experimental canine myocardial reperfusion injury by a monoclonal antibody (anti-Mol, Anti-CDI lb) that inhibits leukocyte adhesion. $J$ Clin Invest 1988;81:624-629.

38. Simpson PJ, Todd RF, Mickelson JK, Fantone JC, Gallagher KP, Tamura Y, Lee KA, Kitzen JM, Lucchesi BR. Sustained limitation of myocardial reperfusion injury by a monoclonal antibody that inhibits leukocyte adhesion. Fed Proc 1988;2:A1237.

39. Mullane KM, Read N, Salmon JA, Moncada S. Role for leukocytes in acute myocardial infarction in anesthetized dogs: relationship to myocardial salvage by antiinflammatory drugs. $J$ Pharm Exp Ther 1984;228:510-552.

40. Flynn PJ, Becker WK, Vercellotti GM, Weisdorf DJ, Craddock PR, Hammerschmidt DE, Lillehei RC, Jacolb H. Ibuprofen inhibits granulocyte responses to inflammatory mediators. Inflammation 1984;8:33-44.

41. Romson JL, Hook BG, Rigot VH, Schork MA, Swanson DP, Lucchesi BR The effect of ibuprofen on accumulation of indium-111-labeled platelets and leukocytes in experimental myocardial infarction. Circulation 1982,66:1002 1011.

42. Arnaout MA, Lanier LL, Faller DV. Relative contribution of the leukocyte molecules Mol, LFA-1, and pl 50,95 (leuM5) in adhesion of granulocytes and monocytes to vascular endothelium is tissue-and stimulus specific. $J$ Cell Physiol 1988; 137:305-309.

43. Simpson PJ, Fantone JC, Mickelson JK, Gallagher KP, Lucchesi BR. Identification of a time window for therapy to reduce experimental canine myocardial injury: suppression of neutrophil activation during 72 hours of reperfusion. Circ Res 1988,63:1070-1079.

44. Jolly SK, Kane WJ, Bailie MB, Abrams GD, Lucchesi BR. Canine myocard al reperfusion injury: its reduction by the combined administration of superoxide dismutase and catalase. Circ Res 1984:54:277-285.

45. Tamura Y, Chi I, Driscoll E, Jr, Hoff PT, Freeman BA, Gallagher KP Lucchesi BR. Superoxide dismutase conjugated to polyethylene glycol provides sustained protection against myocardial ischemia/reperfusion injury in canine heart Circ Res 1988.63.944-959.

46. Zweier JL, Kuppusamy P, Lutty GA. Measurement of endothelial cell free radical generation: evidence for a central mechanism of free radical injury in postischemic tissues. Proc Natl Acad Sci USA 1988;85:4046-4050.

47. Engerson ID, McKelvev $\mathrm{IG}$, Jones HP. Xanthine dehydrogenase to xanthine oxidase conversion in ischemic rat tissues. Fed Proc 1989:3:A628.

48. Hoey BM, Butler J, Halliwell B. On the specificity of allopurinol and oxypurinol as inhibitors of xanthine oxidase. $\Lambda$ pulse radiolysis determination of rate constants for reaction of allopurinol and oxypurinol with hydroxyl radicals. Free Rad Res Comms 1988:4:259-263.

49. Reimer KA, Jennings RB. Failure of the xanthine oxidase inhibitor allopurinol to limit infarct size after ischemia and reperfusion in dogs. Circulation 1985,71:1069-1075

50. Werns SW, Shea MJ, Mitsos SE, Dysko RC, Fantone JC, Schork MA Abrams GD, Pitt B, Lucchesi BR. Reduction of the size of infarction by allopurinol in the ischemic-reperfused canine heart. Circulation 1986;73.518-524. 51. Werns SW, Grum CM, Ventura A, Lucchesi BR. Effects of allopurinol or oxypurinol on myocardial reperfusion injury. Circulation 1987;76:IV-97. 
52. Betts WH, Cleland LG, Gee DJ, Whitehouse MW. Effects of D-penicillamine on a model of oxygen-derived free radical mediated tissue damage. Agents and Actions 1984;283-290.

53. Devi P. Chemical radiation protection by alpha-mercaptopropionyl glycine. $J$ Nucl Med Allied Sci 1983;27:327-336.

54. Milsos SE, Funtone JC, Gallaghter KP, Walden KM, Simpsun PJ, Abrams GD, Schork MA, Lucchesi BR. Canine myocardial reperfusion injury: protection by a free radical scavenger, N-2-mercaptopropionyl glycine. $J$ Cardiovas Pharmacol 1986;8:978-988

55. Myers ML, Bolli R, Lekich RF, Hartley CJ, Roberts R. Enhancement of recovery of myocardial function by oxygen free-radical scavengers after reversible regional ischemia. Circulation 1985:72:915-921

56. Myers ML, Bolli R, Lekich RF, Hartley CJ, Roberts R. N-2-mercaptopropionylglycine improves recovery of myocardial function following reversible regional ischemia. JACC 1986;8:1161-1168.

57. Westlin W, Mullane K. Does captopril attenuate reperfusion-induced myocardial dysfunction by scavenging free radical? Circulation 1988;77:suppl 1:1-30I-39.
58. McCord JM. Oxygen-derived free radicals in post ischemic tissue injury. $N$ Engl J Med 1985;312:159-163.

59. Lucchesi BR, Mullane KM. Leukocytes and ischemia-induced myocardial injury. Ann Rev Pharmacol Toxicol 1986;26:201-224.

60. Werns SW, Lucchesi BR. Leukocytes, oxygen radicals and myocardial injury due to ischemid and reperfusion. Free Radicals Biol Med 1988:4:31-37.

61. Southorn PA, Powis G. Free radicals in medicine: II. Involvement in human disease. Mayo Clinic Proc 1988,63:390-408.

62. Ulraizee A, Reimer KA, Murry CE, Jennings RB. Failure of superoxide dismutase to limit myocardial infarct size in a 40 minutes ischemia/four days reperfusion model in dogs. Circulation 1987:75:1237-1248.

63. Eng C, Cho S, Factor SM, Kirk ES. A nonflow basis for the vulnerability of the subendocardium. JACC 1987,9:374-379.

64. Brown EJ Jr, Mannisi JA, Lillis OI, Cohn PF. Time course of reperfusion induced reversal of early myocardial expansion. JACC 1989;13:109A.

65. Murry CE, Richard VJ, Jennings RB, Reimer KA. Prolonged reperfusion attenuates the protective effect of preconditioning myocardium with a brief episode of ischemia. Fed Proc 1989;3:A629. 